\title{
26166 - NON-STEROIDAL ANTI-INFLAMMATORY DRUGS INCREASE INSULIN RELEASE FROM BETA-CELLS BY INHIBITING ATP-SENSITIVE POTASSIUM CHANNEL
}

\author{
Jingxin Li MD, Nina Zhang; Bin, MSc; Qinhua Wang; Wei-Yang Lu, PhD \\ Sunnybrook And Women's, Toronto, ON, Canada
}

INTRODUCTION: Non-steroidal anti-inflammatory drugs (NSAIDs) are some of the most commonly prescribed medications. NSAIDs effectively reduce chronic pain and inflammation responses via inhibiting cyclooxygenase (COX)(1). Alternatively NSAIDs induce hypoglycemia(2). This study intended to test a hypothesis that NSAIDs decrease blood glucose by increasing insulin secretion.

METHODS: In presence and absence of the NSAID meclofenamic acid (MFA) insulin release from the INS- 1 cell, a widely used â;-cell line, was analyzed using a RIA kit. Perforated patch clamp electrophysiology was used to study the actions of NSAIDs on ion channel functions.

RESULTS: At clinical concentrations, MFA significantly increased insulin release from INS- 1 cells at low but not high glucose. Accordingly MFA depolarized the cell at low but not high glucose by blocking the ATP-gated K+ channel (KATP). MFA blocked KATP through an extracellular interaction and was not involved in intracellular COX inhibition. Moreover, other NSAIDs including acetylsalicylic acid, flufenamic acid and ibuprofen also inhibited KATP in the cell.

DISCUSSION: We reason that NSAIDs inhibit the KATP, thus depolarizing â;-cells, triggering insulin release and consequently lowering blood glucose.

REFERENCES:

1.Pharmacol Ther 2005 Aug; 107(2):139-54.

2.Metabolism 1979 Sep;28(9):887-9 